\title{
Resultados a 10 años de la artrodesis lumbar circunferencial medidos por la escala de Oswestry.
}

\author{
DOI: http://dx.doi.org//10.37315/SOTOCAV20202815517 \\ ORTS-GARCÍA J (1), AVELLANA-ZARAGOZA JA (2), BAS-HERMIDA T (3) \\ (1) SERVICIO DE TRAUMATOLOGÍA, HOSPITAL UNIVERSITARIO DE LA RIBERA. ALZIRA. \\ (2) SECCIÓN DE GERIATRÍA. HOSPITAL UNIVERSITARIO DE LA RIBERA, ALZIRA. \\ (3) UNIDAD DE RAQUIS. HOSPITAL UNIVERSITARIO Y POLITÉCNICO LA FE. VALENCIA.
}

\begin{abstract}
Resumen.
La artrodesis circunferencial se postula como el tratamiento quirúrgico de elección en algunas patologías degenerativas y discales de la columna lumbar, tanto en cirugía primaria como sobre todo en la de revisión. Objetivo: Medir el dolor y la funcionalidad mediante la escala de ODI, en pacientes con dolor lumbar y/o radicular a los que se les ha realizado un abordaje amplio quirúrgico transforaminal junto con la artrodesis anterior y posterior (TLIF). Material y métodos: En nuestra serie intervenimos a 38 pacientes durante los años 2.000-2.003 mediante la técnica TLIF (fusión intersomática transforaminal lumbar) y evaluamos los resultados a largo plazo, diez años, mediante el Oswestry Disability Index (ODI). Resultados: Muestran mejoría en el ODI al comparar las cifras basales, previas a la intervención, con el seguimiento a 10 años $(34,21+/-9,31$ a 11,82+/-7,62 puntos, $p=0,000)$. Esta mejoría también se observa en cada uno de los apartados del ODI de forma significativa: intensidad del dolor, cuidados personales, capacidad para incorporarse, caminar, sentarse, estar de pie, sueño, vida sexual, vida social y viajes ( $p=0,000$, en todos ellos). Conclusión: En nuestra serie de casos, en pacientes con dolor lumbar y/o radicular, la utilización de la técnica TLIF puede haber colaborado a una importante mejora en la puntuación del test al mejorar el dolor, el sueño, la funcionalidad y aspectos sociales, de forma mantenida al menos hasta los 10 años de seguimiento.
\end{abstract}

\section{Summary.}

Circumferential arthrodesis is postulated as the surgical treatment of choice in some de-generative and disc diseases of the lumbar spine, both in primary surgery and especially in revision surgery. Objective: To measure pain and functionality using the ODI scale, in patients with low back and / or radicular pain who have had a comprehensive transforaminal surgical ap-proach together with anterior and posterior arthrodesis (TLIF). Material and methods: In our series, we performed TLIF(transforaminal interbody fusión lumbar) on 38 patients during 2-000-2.003 years and evaluated the longterm results, ten years, using the Oswestry Disability Index (ODI). Results: They show improvement in ODI by comparing the baseline figures, prior to the intervention, with the 10-year follow-up $(34.21+/-9.31$ to $11.82+/-7.62$ points, $p=0.000)$ . This improvement is also observed in each of the ODI sections in a significant way: pain intensity, personal care, ability to get up, walk, sit, stand, sleep, sex life, social life and travel ( $p=0.000$, in all of them).Conclusion: In our case series, in patients with low back and / or radicular pain, the use of the TLIF technique may have contributed to a significant improvement in the test score by improving pain, sleep, functionality and social aspects, maintained at least up to 10 years of follow-up.

Palabras Clave: TLIF, Transforaminal interbody fusion., Artrodesis circunferencial lumbar,

ODI, Oswestry disability Index, Dolor lumbar, Discapacidad. Funcionalidad.

Correspondencia:

Javier Orts García

Ctra. Corbera Km 1, 46600- Alzira

ejortsg@gmail.com 
ORTS J, Y COLS. Resultados a 10 años de la artrodesis lumbar circunferencial, medidos por la escala de Oswestry.

\section{Introducción}

El número de fusiones lumbares está creciendo continuamente en los países desarrollados. Diversas técnicas quirúrgicas con diferentes vías de abordaje, diferente instrumentación y varios materiales para el injerto están en discusión. La artrodesis intersomática de la columna lumbar con cajas metálicas o de fibra de carbono rellenas de hueso son un tratamiento efectivo para el dolor lumbar. En combinación con tornillos pediculares se proporciona un inmediato soporte estructural y una alta tasa de fusión. Además la resección del disco como potencial causa de dolor es crucial para eliminar el dolor de columna discogénico.

El tratamiento quirúrgico de la patología de la columna lumbar mediante abordaje transforaminal unilateral para la fusión intersomática lumbar (TLIF) como alternativa a la fusión intersomática por vía anterior (ALIF), y a la tradicional fusión intersomática posterior (PLIF) ha ganado popularidad en los últimos años.

Los nuevos métodos de medición de los resultados tras la realización de una técnica quirúrgica están basados en cuestionarios que miden los síntomas y el estado funcional relatado por el paciente. Como el dolor de espalda es un síntoma subjetivo, la medición de los resultados abarca lógicamente estas dimensiones subjetivas. Varios de los instrumentos disponibles para medir el estado de salud y el estado funcional utilizados son:

- SF- $36^{1}$ : Realiza una medición genérica del estado de salud.

- Disability Scale de Roland y Morris ${ }^{2}$. Se usa en atención primaria.

- Low Back Pain Disability Questionarie de Oswestry $(\mathrm{ODI})^{3}$. Se usa para estudiar a los pacientes quirúrgicos con un grado alto de validez.

Estos dos últimos están diseñados para medir el estado funcional concreto en el caso de dolor de espalda y tienen la ventaja de captar con mayor detalle cualquier disfunción relacionada con la espalda. ODI valora 10 aspectos: intensidad del dolor, cuidados personales, capacidad para incorporarse, caminar, sentarse, estar de pie, sueño, vida sexual, vida social y viajes, con 6 niveles de puntuación. Su valor final va de 0 a 100 .

La hipótesis que se plantea es que el abordaje amplio quirúrgico transforaminal junto con la artrodesis anterior y posterior (TLIF) puede ofrecer buenos resultados a largo plazo en control del dolor y la funcionalidad, en pacientes con dolor lumbar y/o radicular por distintas etiologías.

El objetivo del presente trabajo fue medir el dolor y la funcionalidad mediante la escala de ODI, en pacientes con dolor lumbar y/o radicular a los que se les ha realizado un abordaje amplio quirúrgico transforaminal junto con la artrodesis anterior y posterior (TLIF).

\section{Material y Métodos}

Se trata de una serie de casos en la que se estudiaron 38 pacientes consecutivos que presentan dolor lumbar y/o radicular que les generaba incapacidad, Se obtuvieron datos referentes a la intensidad y distribución del dolor de forma prospectiva y se evaluaron mediante anamnesis extensa y examen clínico detallado sus antecedentes médicos. Se realizó estudio por imagen mediante radiografías anteroposterior y lateral de la columna lumbar pre y postoperatoria. Todos los pacientes fueron sometidos a Resonancia Nuclear Magnética de la columna preoperatoria. Todos los casos fueron intervenidos por el mismo cirujano.

La edad media fue de $44,79+/-15,64$ años y el $52,6 \%$ fueron hombres $(n=20)$, con discopatía primaria o secuelas de intervenciones anteriores. Las cirugías se realizaron entre los años 2000 y 2003, mediante TLIF lumbar a uno, dos y tres niveles (Fig.1).

Se usaron tornillos pediculares y cajas intersomáticas de titanio cilíndricas (DePuy®), rellenas de hueso autólogo del propio paciente. (Fig.1).

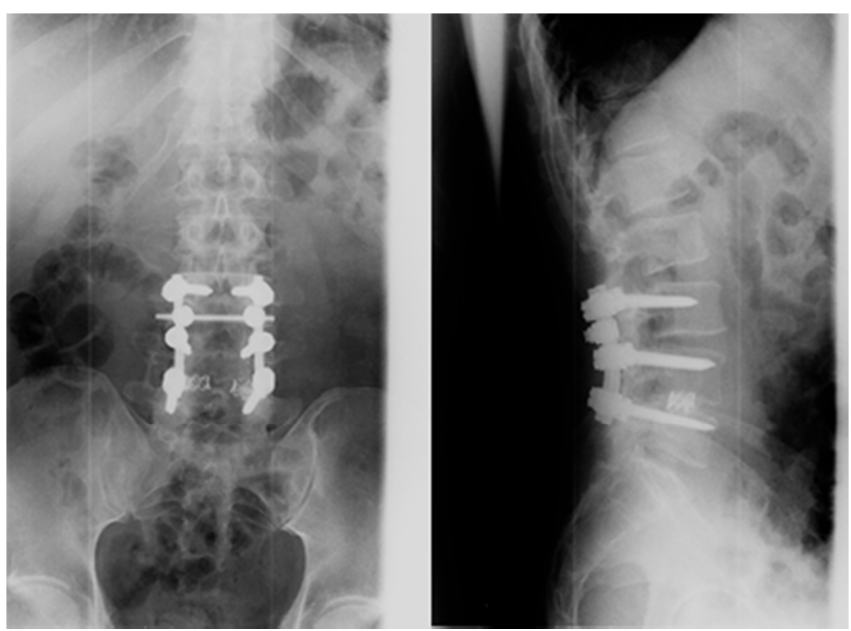

Figura 1. Imágenes de la columna lumbar tras la realización de la técnica TLIF.

Se permitió la deambulación al día siguiente de la cirugía. Se pautó un corsé semirrígido lumbar durante tres meses.

Aplicamos la escala ODI antes de la artrodesis circunferencial (basal) y 10 años después de la misma, valorando los parámetros de dolor y funcionalidad de la escala en un seguimiento a largo plazo.

La toma de datos fue realizada por dos investigadores en todos los pacientes, tanto en la situación basal como en el seguimiento a los 10 años.

Se comprobó si los resultados de las distintas variables seguían una distribución normal (Shapiro-Wilk). En los casos que sí tenían una curva de distribución normal se utilizaron test estadísticos paramétricos para la comparación de variables cuantitativas, $t$ de Student para muestra apareadas. En los casos en los que no seguían 
ORTS J, Y COLS. Resultados a 10 años de la artrodesis lumbar circunferencial, medidos por la escala de Oswestry.

una distribución normal se han utilizado test no paramétricos: Wilcoxon.

El estudio fue aprobado por la Comisión de Investigación de nuestro Centro.

\section{Resultados}

No se presentó ninguna complicación ni secuela tras la cirugía en los 38 pacientes estudiados, excepto en dos casos que precisaron la ampliación de la artrodesis a nivel proximal. La distribución por diagnósticos fue: espondilolistesis $(n=10)$, secuelas postdiscectomía $(n=12)$, enfermedad discal $(n=9)$ y pseudoartrosis $(n=7)$.

Los resultados en la escala ODI se muestran en la Figura 2, donde se observa una disminución significativa de casi un $70 \%$ en la puntuación a los 10 años de seguimiento, respecto a la situación basal previa a la intervención mediante técnica TLIF $(34,21+/-$ 9,31 a $11,82+/-7,62$ puntos, intervalo de confianza $95 \%$ : 18.85-25.94; $p=0,000)$. Los resultados basales y a los 10 años siguen ambos una distribución normal, mientras que para los distintos apartados del ODI se ha utilizado una distribución no paramétrica por no ajustarse a la normal (Tabla I).

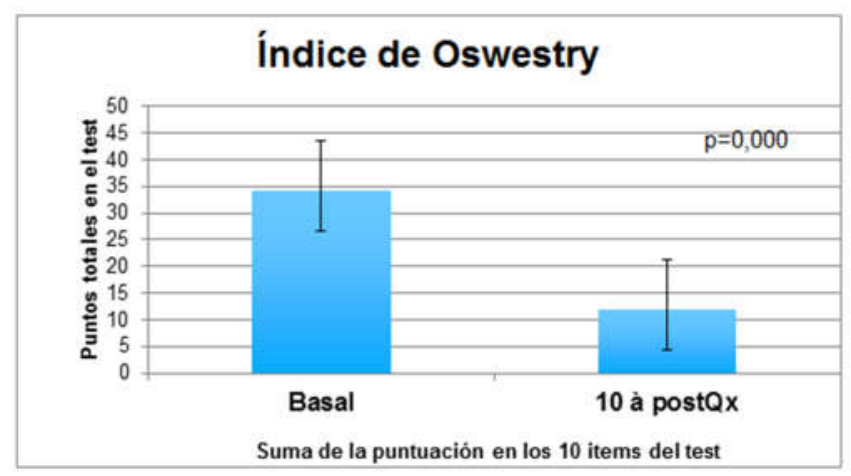

Figura 2. Puntuación media del ODI antes y 10 años tras la cirugía.

\begin{tabular}{|l|l|l|l|l|}
\hline Test de Oswestry & Basal (n) & $\begin{array}{l}10 \text { años postci- } \\
\text { rugia (n) }\end{array}$ & $\begin{array}{l}\text { Diferencia } \\
(\%)\end{array}$ & $\begin{array}{l}\text { Z Wilcoxon / } \\
\text { Significación }\end{array}$ \\
\hline Intensidad Dolor & $3,87+/-0,84(38)$ & $1,11+/-1,01(38)$ & $-71,3 \%$ & $Z=5,34 / P=0,000$ \\
\hline $\begin{array}{l}\text { Actividad Vida co- } \\
\text { tidiana }\end{array}$ & $3,13+/-1,47(38)$ & $0,76+/-0,91(38)$ & $-75,7 \%$ & $Z=5,05 / P=0,000$ \\
\hline Levantar objetos & $3,26+/-1,59(38)$ & $1,45+/-1,29(38)$ & $-55,5 \%$ & $Z=3,96 / P=0,000$ \\
\hline Caminar & $3,74+/, 1,33(38)$ & $0,95+/-0.96(38)$ & $-74,6 \%$ & $Z=5,27 / P=0,000$ \\
\hline Sentarse & $3,29+/-1,71(38)$ & $0,97+/-1,10(38)$ & $-70,5 \%$ & $Z=4,14 / P=0,000$ \\
\hline Pararse & $3,76+/-1,38(38)$ & $1,61+/-1,44(38)$ & $-57,2 \%$ & $Z=4,76 / P=0,000$ \\
\hline Dormir & $3,80+/-1,45(38)$ & $0,95+/-0,99(38)$ & $-75,0 \%$ & $Z=5,06 / P=0,000$ \\
\hline Actividad Sexual & $3,50+/-1,44(32)$ & $1,66+/-1,23(32)$ & $-52,6 \%$ & $Z=4,10 / P=0,000$ \\
\hline Actividad Social & $3,71+/-1,25(38)$ & $1,66+/-1,21(38)$ & $-55,3 \%$ & $Z=5,05 / P=0,000$ \\
\hline Viajar & $3,50+/-1,72(38)$ & $0,97+/-0,97(38)$ & $-72,3 \%$ & $Z=-4,96 / P=0,000$ \\
\hline
\end{tabular}

Tabla I. Puntuación de los distintos apartados del ODI basal y a los 10 años de la cirugía y significación del cambio de puntuación en cada uno de los componentes.

\section{Discusión}

Hay abundantes ejemplos en la literatura en los que la asociación de los síntomas que presentan los pacientes con los resultados funcionales es muy débil ${ }^{1}$ : la reducción de la movilidad en la columna puede estar asociada a una mejora del dolor y la incapacidad, o a un descenso en el riesgo de sufrir dolor; los antidepresivos tricíclicos disminuyen el dolor y la depresión, no modifican la concentración de beta-endorfinas en liquido cefaloraquídeo, y no modifican la actividad electromiográfica paravertebral; las correlaciones entre la movilidad de la columna lumbar y el Índice de Oswestry modificado, solo son de 0.04 a 17 (valor absoluto).

En la artrodesis posterolateral del raquis la mayoría de autores coinciden en que la instrumentación pedicular con auto injerto a nivel posterior es el sistema de osteosíntesis más estable ${ }^{4}$. Sin embargo es en la artrodesis intersomática donde se aplican los principios fundamentales de la osteosíntesis: neutralizaciónsostén mediante placas o barras solidarizadas a tornillos en los cuerpos vertebrales que proporcionan el principio del tirante, y la compresión-sostén al usar un elemento de estabilización intersomático como un autoinjerto cortical ${ }^{4,5,6}$. Por tanto, junto al constructo mecánico, es necesario un aporte biológico que consiga la fusión tutelada ya que, de lo contrario, el material acaba rompiéndose por fatiga y la instrumentación fracasa. Por ello en algunos casos se combina la fijación posterolateral con la anterior, lo que se conoce como artrodesis en $360^{\circ}$. De este modo, en cualquier acción terapéutica la planificación biomecánica y biológica deben ir juntas para que el objetivo del tratamiento se alcance con éxito. La Fusión Lumbar Intersomática Transforaminal (TLIF) fue utilizada por Harms en 191 pacientes entre 1993 y 1996 y publicados sus resultados en $1.998^{8}$. Las indicaciones establecidas son espondilolistesis degenerativa o ístmica, dolor lumbar discogénico y síndromes postdiscectomía rebeldes al tratamiento conservador. Uno de sus objetivos es evitar algunos de los inconvenientes de la Fusión Lumbar Intersomática Posterior (PLIF), tales como la excesiva retracción de la raíz nerviosa, (necesaria para introducir el injerto intersomático), la aracnoiditis y la fibrosis peridural ${ }^{9}$. Otra ventaja con respecto a ALIF es evitar el abordaje a la columna trans o retroperitoneal, ya que está asociado a eyaculación retrograda, lesión de grandes vasos y a un periodo de recuperación más largo en el caso de realizarla en dos tiempos.

Por otra parte en los últimos tiempos se ha incrementado la presión ejercida sobre las responsabilidades en el sistema de asistencia médica, la comprobación de la eficacia de todo lo que hacemos y la justificación de los elevados costes que genera la atención médica. Los economistas de la salud a menudo señalan que no tiene por qué existir una relación directa entre los costes de la asistencia y su 
ORTS J, Y COLS. Resultados a 10 años de la artrodesis lumbar circunferencial, medidos por la escala de Oswestry.

calidad. Los estudios realizados en Maine dejaron entrever que en el caso de las intervenciones quirúrgicas para las hernias discales y las estenosis lumbares, los resultados eran superiores cuantas menos intervenciones y peores cuanto mayor número de ellas se realizaban ${ }^{10}$.

Nuestro estudio ha evaluado la puntuación global y cada uno de los aspectos relacionados con la misma y medidos mediante el ODI en situación basal, antes de la intervención, y a los 10 años de la misma. Por tanto se han analizado el dolor y algunos aspectos relacionados, la capacidad para realizar actividades de la vida diaria y aspectos sociales, que a su vez están todos ellos relacionados con la calidad de vida ${ }^{1}$. En la situación basal los pacientes presentaban un grado de discapacidad moderada a severa (34,2+/-9,3; puntuación entre $20-40 \%$ se considera moderada) y a los 10 años de la intervención mostraban una discapacidad mínima $(11,8+/-7.6$, puntuación entre 0 $20 \%$ se considera mínima) ${ }^{1}$. Éste instrumento pese a ser el más utilizado en la evaluación de la funcionalidad en pacientes intervenidos de patología de columna, ha sido criticado al no representar los distintos tipos de alteración funcional que pueden tener los sujetos sometidos a más de tres niveles de fusión lumbar y edad avanzada (>70 años), al año y tres años de seguimiento ${ }^{11}$.

La disminución del porcentaje de discapacidad antes de la cirugía comparado con el posterior a la misma, en un seguimiento medio postoperatorio de 10 años, ha sido de más del $50 \%$, concretamente 22,4 puntos (un $65,4 \%$ ) de forma significativa $(p=0,000)$. Además la mejoría en la puntuación total del test se acompaña de la mejoría en cada uno de los 10 aspectos evaluados, tanto el de dolor, como los de movilidad, actividades de la vida diaria, sueño y aspectos sociales (ver tabla I), por lo que podemos decir que la mejoría en la puntuación en el ODI se ha obtenido al mejorar el dolor, el sueño y la funcionalidad de forma muy importante y a largo plazo.

No hay estudios previos realizados de seguimiento a tan largo plazo en la bibliografía consultada, el seguimiento de más tiempo ha sido los recientes estudios de Boktor (2018) de dos años ${ }^{12}$ y el de Liow (2019) de 5 años de seguimiento $^{13}$. En esta última publicación se realiza la técnica MIS-TLIF (TLIF mínimamente invasivo) y se aplica el ODI a los cinco años. Concluye que los adultos con peor función basal en el test eran los que más tiempo tardaban en regresar al trabajo, no habiendo diferencias en función de la edad ni en el índice de masa corporal, ni en la prevalencia de comorbilidad. Otro estudio de seguimiento a tres años que también analiza la funcionalidad mediante el ODI pero solo en pacientes intervenidos para la corrección de la deformidad de columna del adulto, publica que un $72 \%$ de los pacientes intervenidos por ésta causa mantienen una buena función a los 3 años y ello depende de la edad (peor los mayores), de la fragilidad, la comorbilidad preoperatoria (osteoporosis, hipertensión, patología pulmonar) y la vía de abordaje quirúrgico (peor resultado en la posterior) ${ }^{14}$. En un estudio retrospectivo que analiza la eficacia de la cirugía endoscópica transforaminal percutánea en pacientes con hernia discal lumbar migrada, refieren mejoría en el ODI a los 12 meses de seguimiento de 62,4+/-8,2 a $9,2+/-3,4^{15}$. Como hemos comentado no hay estudios con cuestionarios estandarizados para conocer los resultados a largo plazo, más allá de los 5 años.

En nuestra serie solo presentaron complicación tras la cirugía dos casos de los 38 pacientes estudiados, que precisaron la ampliación de la artrodesis a nivel proximal. Más recientemente a la realización de nuestro estudio se ha generalizado el uso del MIS-TLIF como técnica para disminuir algunas complicaciones que pueden aparecer en el PLIF y que puedan condicionar reintervenciones y empeoramiento del pronóstico ${ }^{16}$.

En una revisión sistemática y metanálisis ${ }^{17}$ de nueve estudios incluyendo a 990 pacientes se compara TLIF frente a PLIF y concluyen que el TLIF supera al PLIF en menor tasa de complicaciones, menor pérdida sanguínea y menor tiempo de cirugía. El resultado clínico es similar con una ligera mejoría del ODI postoperatorio para el TLIF. En nuestro estudio la mejoría ha sido global, incluso en los dos pacientes que precisaron reintervención, mejorando en todos los aspectos funcionales, sin embargo los apartados que mayor mejoría han presentado en nuestro estudio han sido la capacidad para realizar las actividades de la vida diaria, la deambulación, el sueño, la capacidad para viajar y el dolor.

\section{Conclusiones}

En nuestra serie de casos, en pacientes con dolor lumbar y/o radicular, la utilización de la técnica TLIF puede haber colaborado a una importante mejora en la puntuación del Índice de Discapacidad de Oswestry al mejorar el dolor, el sueño, la funcionalidad y aspectos sociales, de forma mantenida al menos hasta los 10 años de seguimiento. 
ORTS J, Y COLS. Resultados a 10 años de la artrodesis lumbar circunferencial, medidos por la escala de Oswestry.

\section{Bibliografía}

1. Chapman JR, Norwell DC. Evaluating Common Outcomes for Measuring Treatment Success for Chronic Low Back Pain. Spine 2011; 36(21):S54-S68.

2. Roland M, Morris R. A study of the natural history of back pain: I. Development of a reli-able and sensitive measure of disability in low back pain. Spine 1983; 8:141-4.

3. Fairbank JCT, Davies JB, Mbaot JC, O'brien JT. The Oswestry Low Back Pain Disability Questionnarie. Physiotherapy 1980; 66:271-3.

4. Guerado E. Osteogénesis terapéutica en cirugía del raquis. Bases científicas de la ar-trodesis vertebral I: Fundamentos biomecánicos. Rev. Ortop. Traum 2005; 49:29-45

5. Guerado E, Fuerstenberg $\mathrm{CH}$. What bone graft substitutes should we use in posttrau-matic spinal fusion? Injury 2011; 42 Suppl 2:S64-71.

6. Guerado E, Godino M, Andrades JA, Becerra J. Osteogénesis terapéutica en cirugía del raquis II. Fundamentos biológicos. Rev Ortop Traum 2005; 49:46-58.

7. Schofferman J, Slosar P, Reynolds J, Goldthwaite N, Whiteah I, Keaney D. A prospec-tive randomized comparison of 270 degrees fusions to 360 degrees fusions (circumferen-tial fusions). Spine 2001; 26:E207-212.

8. Harms J, Jeszenszky D. The unilateral transforaminal approach for posterior lumbar interbody fusion. Orthop Traumatol 1998; 6:88-99.

9. Wetzel FT, LaRocca H. The failed posterior lumbar interbody fusion. Spine $1991 ; 16: 839-845$.

10. Atlas SJ, Deyo RA, Keller RB, Chapin AM, Patrick DL, Long JM, Singer DE. The Maine Lumbar Spine Estudy, Part II. 1-year outcomes of surgical and non-surgical management of sciatica. Spine 1996, 21:1777-86.

11. Park JS, Lee CS, Park SJ, Lee KJ, Yum TH. Minimum Three-Year Follow-up of Specif-ic Functional Disabilities After Multi-Level Lumbar Fusion: Comparison of Long-Level and Short-Level Fusion. Spine 2019; 29. doi: 10.1097/BRS.0000000000003093.

12. Boktor JG, Pockett RD, Verghese N. The expandable transforaminal lumbar interbody fusion - Two years followup. J Craniovertebr Junction Spine 2018;9: 50-5.

13. Liow MHI, Goh GS, Yeo W, Ling ZM, Yue WM, Guo CM, Tan SB. Time Taken to Re-turn to Work Does Not Influence Outcomes of Minimally Invasive Transforaminal Lumbar Interbody Fusion: A 5-Year Follow-Up Study. Spine 2019; 44:503-9.

14. Passias PG, Bortz CA, Lafage V, Lafage R, Smith JS, Line B, Eastlack R, Gupta MC, Hostin RA, Horn SR, Segreto FA, Egers M, Sciubba DM, Gum JL, Kebaish KM, Klineberg EO, Burton DC, Schwab FJ, Shaffrey Cl, Ames CP, Bess S. Durability of Satisfactory Functional Outcomes Following Surgical Adult Spinal Deformity Correction: A 3-Year Survivor-ship Analysis. Oper Neurosurg (Hagerstown) 2019; 31:pii:opz093. doi: 10.1093/ons/opz093.

15. Yao Y, Qin R, Feng Q, Jiang X, Zhou P, Guo Z, Zhang F. Percutaneous Endoscopic Transforaminal Decompression in the Treatment of Patients with Migrated Lumbar Disc Herniation: A Retrospective Study. World Neurosurg 2019; 29:S1878-8750.

16. Lin GX, Park CK, Hur JW, Kim JS. Time Course Observation of Outcomes between Minimally Invasive Transforaminal Lumbar Interbody Fusion and Posterior Lumbar Inter-body Fusion. Neurol Med Chir (Tokyo) 2019; 9. doi: 10.2176/nmc.oa.2018-0194.

17. De Kunder SL, van Kuijk SMJ, Rijkers K, van Hemert WLW, de Bie RA, van Santbrink H. Tramsforaminal lumbar interbody fusión (TLIF) versus posterior lumbar interbody fusión (PLIF) in lumbar espondylolisthesis: a systematic review and meta- analysis. Spine 2017; 11:1712-21. 